\title{
LA.P08
}

\section{Crystal structure of the complex of GLPO with an anti-thyroid drug PTU}

\author{
A. Bhushan ${ }^{1}$, R. Singh ${ }^{1}$, A. Singh ${ }^{1}$, M. Sinha ${ }^{1}$, P. Kaur ${ }^{1}$, S. Sharma ${ }^{1}$, T. Singh $^{1}$ \\ ${ }^{1}$ Department of Biophysics AlIMS, India
}

Lactoperoxidase is a member of mammalian heme peroxidase superfamily that consists of lactoperoxidase (LPO), myloperoxidase (MPO), thyroid peroxidase (TPO), eosinophil peroxidase (EPO). So far crystal structures of only two mammalian peroxidases LPO and MPO have been determined. The structures of LPO have been determined from the samples obtained from bovine, buffalo, goat and sheep. Their complexes have also been determined with inhibitors and substrate analogues. However, the structures of EPO and TPO have not been obtained so far. Due to significant sequence identity between LPO and TPO, the structures of these proteins are expected to be similar. Similarly their substrates will also have similarity as well as the substrate and enzyme interactions will be similar. Therefore, a complex of LPO was prepared with propylthiouracil (PTU), a compound used as a drug in thyroid ailments. The complex was crystallized using co-crystallization. The crystals of native LPO were also soaked in the solution containing PTU. The crystals belonged to monoclinic space group with cell dimensions $a=80.2 \AA, b=82.5, c=95.0, \beta=73.70$ There were four molecules of LPO in the asymmetric unit. The structure determination of the complex revealed that PTU binds to the LPO at the distal heme site. It is held at this site through several hydrogen bonds and van der Waals contacts. The mode of binding and number of interactions suggest that a similar mode of binding may occur with TPO. Based on the information of interactions and the missing potential interactions, the modifications in the structure of PTU are suggested so that the improved design of the TPO inhibitor is obtained.

[1] Singh, A. K., Singh, N., Sharma, S., Singh, S. B., Kaur, P., Bhushan, A., Srinivasan, A., and Singh, T. P. (2008)J. Mol. Biol. 376, 1060-1075, [2] Singh, A. K., Singh, N., Sinha, M., Bhushan, A., Kaur, P., Srinivasan, A., Sharma, S., and Singh, T. P. (2009) J. Biol. Chem. 284, 20311-20318, [3] Sheikh, I. A., Singh, A. K., Singh, N., Sinha, M., Singh, S. B., Bhushan, A., Kaur P., Srinivasan, A., Sharma, S., and Singh, T. P. (2009) J. Biol. Chem. $284,14849-14856$

Keywords: GLPO, Anti-Thyroid, propyl thiouracil 